\title{
Pricing Basket Options by Polynomial Approximations
}

\author{
Pablo Olivares and Alexander Alvarez \\ Department of Mathematics, Ryerson University, 350 Victoria St., Toronto, ON, Canada M5B 2K3 \\ Correspondence should be addressed to Pablo Olivares; pablo.olivares@ryerson.ca
}

Received 2 May 2016; Revised 21 July 2016; Accepted 26 July 2016

Academic Editor: Turgut Öziş

Copyright (C) 2016 P. Olivares and A. Alvarez. This is an open access article distributed under the Creative Commons Attribution License, which permits unrestricted use, distribution, and reproduction in any medium, provided the original work is properly cited.

We propose a closed-form approximation for the price of basket options under a multivariate Black-Scholes model. The method is based on Taylor and Chebyshev expansions and involves mixed exponential-power moments of a Gaussian distribution. Our numerical results show that both approaches are comparable in accuracy to a standard Monte Carlo method, with a lesser computational effort.

\section{Introduction}

The objective of the paper is the study of the pricing of basket options under a multivariate Black-Scholes model, using two different polynomial approximations of a related conditional contract.

Our main contribution consists in the proposal of an efficient methodology that combines two approaches previously considered. First, we compute the conditional expected value of the payoff with respect to some of the underlying assets in order to reduce the problem to one-dimensional pricing; see [1]. Then, we use Taylor and Chebyshev polynomials to approximate the resulting conditional price. Numerical findings show that, within an equivalent accuracy, both approximations considerably reduce the computational time necessary to obtain the price, when compared with a standard Monte Carlo method.

While a Taylor expansion of the second order for spread pricing has been considered in [2], an approach based on Chebyshev expansion remains less explored. Recently, it has been considered in [3] for a single-asset option contract and in a multiasset setting in [4]. The latter combines with a Fourier series development, offering an interesting analysis of the error in the approximation. Our method conceptually differs from the works cited above in the way the expansion is carried on.

In addition, our expansions are presented in a general setting with the purpose of illustrating applications in higher dimensions, to other contracts and models, at the expense of somehow more complicated notations.

Basket options are multivariate extensions of univariate European calls or puts. A call basket option takes the weighted average of a group of $d$ stocks (the basket) as the underlying asset and produces a payoff equal to the maximum of zero and the difference between the weighted average and the strike (or the opposite difference for the case of a put). Financial indices such as the S\&P 500 and real options based on the difference between gas and oil prices are examples of such contracts.

In the case of European spread options, whose payoffs are given in terms of the spread of both prices at maturity, several approximations have been considered in the works of $[2,5-10]$, among others, where different ad hoc approaches are studied.

The approach to pricing by Taylor expansions can be traced back to [11], where a method to the price of onedimensional derivatives is proposed. See also [12] for the use of Taylor in valuation of basket and Asian options, where the expansion is made about the characteristic function. Furthermore, in [10] the Taylor expansion is compared with other pricing techniques, proving to be effective and accurate for most values in the parametric space. In our case, the expansion is done on the function resulting from the conditional price, as opposed to a development based on the conditional strike price, as previously considered. Moreover, our methodology hinges on the calculation of mixed exponentialpower moments of a Gaussian distribution and is extended to 
expansions about any point and order. Our point of view may allow for a better control on the approximation.

Taylor expansions produce reasonable approximations in terms of a simple closed-formula. Nonetheless, they are very sensible to the point around which the development is done. Moreover, as this expansion is local, it may introduce significant errors, albeit infrequent, at values far from the point where the expansion is considered, typically the conditional average price under the risk neutral measure. Significant errors for out-of-the-money contracts or extreme values in the parametric set are reported.

In order to overcome this potential problem, we study developments in terms of Chebyshev polynomials, which offer a uniform convergence of the conditional price on a predetermined closed interval. Interesting alternative approximations in terms of Fourier series can be found in $[13,14]$.

The organization of the paper is the following: in Section 2, we introduce the model and main notations and derive a Taylor approximation for $d$-dimensional basket options; in Section 3, we implement Taylor method to spread contracts while in Section 4 we study the case of a Chebyshev approximation and the sensitivity with respect to the spot prices; in Section 4, we discuss the numerical implementation and results; finally, in Section 5 we conclude.

\section{Basket Derivatives and Taylor Expansions}

We introduce some notations. Let $\left(\Omega, \mathscr{F},\left(\mathscr{F}_{t}\right)_{t \geq 0}, \mathbb{P}\right)$ be a filtered probability space. We define the filtration $\mathscr{F}^{X_{t}}:=$ $\sigma\left(X_{s}, 0 \leq s \leq t\right) \subset \mathscr{F}_{t}$ as the $\sigma$-algebra generated by the random variables $\left\{X_{s}, 0 \leq s \leq t\right\}$ completed in the usual way. Denote by $Q$ an equivalent martingale risk neutral measure (EMM) and by $E_{Q}$ the expectation under Q. Quantities $\mu_{a, b}(k), M_{X}(u)$ and $M_{X}(u, a, b)$ represent, respectively, the truncated $k$ th moment and the moment generating function (m.g.f.) truncated on $[a, b]$, while the function $N(\cdot)$ is the cumulative distribution function (c.d.f.) of a standard normal distribution.

The matrix $A^{\prime}$ represents the transpose of matrix $A=$ $\left(a_{i j}\right)$, while $\operatorname{diag}(A)$ is a column vector with components from the diagonal of the matrix $A$. On the other hand, $A^{1 / 2}$ denotes a matrix such that $A^{1 / 2} A^{1 / 2}=A$. For a $d$-dimensional vector $Y$, denote by $\widetilde{Y}$ the same vector, excluding the first component.

By $r$, we denote the (constant) interest rate or a vector with components equal to $r$. The matrix $I_{d}$ is the $d \times d$ identity matrix.

For $l$-times differentiable function $f$ on $\mathbb{R}^{d}$ and a vector $L=\left(l_{1}, l_{2}, \ldots, l_{d}\right)$ with $l_{k} \in \mathbb{N}$ such that $\sum_{k=1}^{d} l_{k}=l, D^{L} f$ represents its mixed partial derivative of order $l$ differentiated $l_{k}$ times w.r.t. $y_{k}$.

The process of spot prices is denoted by $S=$ $\left(S_{t}^{(1)}, S_{t}^{(2)}, \ldots, S_{t}^{(d)}\right)_{t \geq 0}$ for $0 \leq t \leq T$, while $Y=\left(Y_{t}^{(1)}, Y_{t}^{(2)}, \ldots\right.$, $\left.Y_{t}^{(d)}\right)_{t \geq 0}$ is the asset log-return process; they are related by

$$
S_{t}^{(j)}=S_{0}^{(j)} \exp \left(Y_{t}^{(j)}\right) \text { for } j=1,2, \ldots, d \text {. }
$$

The log-return process follows the dynamic

$$
d Y_{t}=\left(r-\frac{1}{2} \operatorname{diag}(\Sigma)\right) d t+\Sigma^{1 / 2} d B_{t}
$$

where $\left(B_{t}\right)_{t \geq 0}$ is a multivariate standard Brownian motion and $\Sigma$ is a positive definite symmetric matrix with components $\left(\sigma_{i j}\right)_{i, j=1,2, \ldots, d}$, with $\sigma_{i i}=\sigma_{i}^{2}$.

We analyze European Basket options whose payoff at maturity $T$, for a strike price $K$, is given by

$$
h\left(S_{T}\right)=\left(\sum_{j=1}^{d} w_{j} S_{T}^{(j)}-K\right)_{+}
$$

where $\left(w_{j}\right)_{1 \leq j \leq d}$ are some deterministic weights and $x_{+}=$ $\max (x, 0)$.

The main examples are spread options, defined for $d=2$ by a payoff:

$$
h\left(S_{T}\right)=\left(S_{T}^{(1)}-S_{T}^{(2)}-K\right)_{+} .
$$

Other related contracts are the so-called $3: 2: 1$ crack spreads with payoff:

$$
h\left(S_{T}\right)=\left(\frac{2}{3} S_{T}^{(1)}-\frac{1}{3} S_{T}^{(2)}-S_{T}^{(3)}-K\right)_{+}
$$

where $S_{T}^{(1)}, S_{T}^{(2)}$, and $S_{T}^{(3)}$ are, respectively, the spot prices of gasoline, heating oil, and crude oil.

Exchange options are spread options with $K=0$; see [15].

We start writing the price of the basket option, denoted by $C_{B}$, in terms of a conditional price via the following elementary proposition.

Proposition 1. Let $C_{B}$ be the price of a European Basket contract with maturity at $T$, strike price $K$, and payoff $h\left(S_{T}\right)$ under the model given by (1) and (2). Then,

$$
\begin{aligned}
& C_{B}=w_{1} E_{Q}\left[\exp \left(-\left(r-\frac{1}{2} \sigma_{Y_{T}^{(1)} / \widetilde{Y}_{T}}^{2}\right) T+\mu_{Y_{T}^{(1)} / \widetilde{Y}_{T}}\right)\right. \\
&\left.\cdot C\left(\tilde{Y}_{T}\right)\right],
\end{aligned}
$$

where, for $y \in \mathbb{R}^{d-1}$,

$$
C(y):=C_{B S}\left(S_{0}^{(1)}, K(y), r, \sigma_{Y_{T}^{(1)} / \tilde{Y}_{T}=y}, T\right)
$$

is the Black-Scholes price of a call option with maturity at $T>$ 0 , volatility $\sigma_{Y_{T}^{(1)} / \widetilde{Y}_{T}=y}$, spot price $S_{0}^{(1)}$, and strike price

$$
\begin{aligned}
K(y)= & \frac{1}{w_{1}} \exp \left(\left(r-\frac{1}{2} \sigma_{Y_{T}^{(1)} / \widetilde{Y}_{T}=y}^{2}\right) T-\mu_{Y_{T}^{(1)} / \widetilde{Y}_{T}=y}\right) \\
& \cdot\left(K-\sum_{j=2}^{d} w_{j} S_{0}^{(j)} e^{y^{(j)}}\right)
\end{aligned}
$$


with

$$
\begin{aligned}
\mu_{Y_{T}^{(1)}} \widetilde{Y}_{T}= & \left(r-\frac{1}{2} \sigma_{11}\right) T \\
& +\Sigma_{1 \widetilde{Y}} \Sigma_{\widetilde{Y}}^{-1}\left(\widetilde{Y}_{T}-r+\frac{1}{2} \operatorname{diag}\left(\Sigma_{\widetilde{Y}}\right)\right) T, \\
\sigma_{Y_{T}^{(1)} / \widetilde{Y}_{T}}= & \sigma_{11}-\Sigma_{1 \widetilde{Y}} \Sigma_{\widetilde{Y}}^{-1} \Sigma_{1 \widetilde{Y}}^{\prime},
\end{aligned}
$$

where

$$
\Sigma_{1 \widetilde{Y}}=\left(\sigma_{12}, \sigma_{13}, \ldots, \sigma_{1, d-1}\right)
$$

and $\Sigma_{\tilde{Y}}$ is the covariance matrix of the vector $\widetilde{Y}_{T}$.

Proof. From (2),

$$
Y_{T}=\left(r-\frac{1}{2} \operatorname{diag}(\Sigma)\right) T+\sqrt{T} \Sigma^{1 / 2} Z_{d}
$$

in law, where $Z_{d}$ is a random variable with a multivariate standard normal probability distribution in $\mathbb{R}^{d}$. Moreover, independently of $\widetilde{Y}_{T}$, the random variable $Y_{T}^{(1)}$ has a univariate normal distribution. Thus, we can write

$$
Y_{T}^{(1)}=\mu_{Y_{T}^{(1)} / \widetilde{Y}_{T}}+\sqrt{T} \sigma_{Y_{T}^{(1)} / \widetilde{Y}_{T}} Z_{1}
$$

in law, where $Z_{1}$ is independent of $Y_{T}$ and it has, conditionally on $\widetilde{Y}_{T}$, a standard univariate normal distribution. Moreover, it is well known (see, e.g., [16]) that $\mu_{Y_{T}^{(1)} / \widetilde{Y}_{T}}$ and $\sigma_{Y_{T}^{(1)} / \widetilde{Y}_{T}}$ are, respectively, given by (9).

Next, by the iterated property of the conditional expected value, we have

$$
\begin{aligned}
C_{B} & :=e^{-r T} E_{\mathscr{Q}}\left(h\left(S_{T}\right)\right)=e^{-r T} E_{\mathscr{Q}}\left(E_{Q}\left(h\left(S_{T}\right) \mid \mathscr{F}_{T}\right)\right) \\
& =w_{1} e^{-r T} E_{\mathscr{Q}}\left(E _ { \mathscr { Q } } \left[\left(S_{0}^{(1)} \exp \left(Y_{T}^{(1)}\right)\right.\right.\right. \\
& \left.\left.\left.-\left(\frac{K}{w_{1}}-\sum_{j=2}^{d} \frac{w_{j}}{w_{1}} S_{0}^{(j)} \exp \left(Y_{T}^{(j)}\right)\right)\right)_{+} \mid \mathscr{F}^{\tilde{Y}_{T}}\right]\right) \\
& =w_{1} e^{-r T} E_{\mathscr{Q}}\left(E _ { \mathscr { Q } } \left[\left(S_{0}^{(1)} \exp \left(Y_{T}^{(1)}\right)-K_{1}\left(\widetilde{Y}_{T}\right)\right)_{+} \mid\right.\right. \\
& \left.\left.\mathscr{F}^{\tilde{Y}_{T}}\right]\right),
\end{aligned}
$$

where $K_{1}(y)=K / w_{1}-\sum_{j=2}^{d}\left(w_{j} / w_{1}\right) S_{0}^{(j)} e^{y^{(j)}}$.
Moreover, substituting (12) into (13), we have

$$
\begin{aligned}
C_{B} & =w_{1} e^{-r T} E_{\mathscr{Q}}\left[E _ { \mathscr { Q } } \left(\left(S_{0}^{(1)}\right.\right.\right. \\
& \left.\cdot \exp \left(\mu_{Y_{T}^{(1)} / \widetilde{Y}_{T}}+\sigma_{Y_{T}^{(1)} / \widetilde{Y}_{T}} \sqrt{T} Z^{(1)}\right)-K^{\prime}\left(\widetilde{Y}_{T}\right)\right)_{+} \mid \\
& \left.\left.\widetilde{F}_{T}\right)\right]=w_{1} e^{-r T} E_{Q}\left[\operatorname { e x p } \left(-r T+\frac{1}{2} \sigma_{Y_{T}^{(1)} / \widetilde{Y}_{T}}^{2} T\right.\right. \\
& \left.+\mu_{Y_{T}^{(1)} / \widetilde{Y}_{T}}\right) E_{Q}\left(\left(S_{0}^{(1)}\right.\right. \\
& \cdot \exp \left(r T-\frac{1}{2} \sigma_{Y_{T}^{(1)} / \widetilde{Y}_{T}}^{2} T+\sigma_{Y_{T}^{(1)} / \widetilde{Y}_{T}} \sqrt{T} Z^{(1)}\right) \\
& \left.\left.\left.-K_{1}\left(\widetilde{Y}_{T}\right)\right)_{+} \mid \widetilde{F}_{T}\right)\right],
\end{aligned}
$$

where

$$
\begin{aligned}
& C\left(\widetilde{Y}_{T}\right)=e^{-r T} E_{Q}\left[\left(S_{0}^{(1)}\right.\right. \\
& \cdot \exp \left(\left(r-\frac{1}{2} \sigma_{Y_{T}^{(1)} / \widetilde{Y}_{T}}^{2}\right) T+\sigma_{Y_{T}^{(1)} / \widetilde{Y}_{T}} \sqrt{T} Z^{(1)}\right) \\
& \left.\left.\quad-K\left(\widetilde{Y}_{T}\right)\right)_{+} \mid \mathscr{F}_{T}^{\widetilde{Y}_{T}}\right] .
\end{aligned}
$$

Hence, we can recognize in $C\left(\widetilde{Y}_{T}\right)$ the expression of a BlackScholes price with the parameters mentioned above.

Remark 2. Notice that $K(y)$ may be negative. In this case, $C(y)$ is not the Black-Scholes price, but the formula remains valid. Condition $w_{j} \leq 0, j=2, \ldots, d$, guarantees its positiveness, as it is the case of spreads and $3: 2: 1$ crack spread contracts defined above.

Notice that $C(y)$ is smooth enough to apply a $k$ th-order Taylor development around any point $y^{*} \in \mathbb{R}^{d-1}$. After conditioning on the last $d-1$ underlying assets, it leads to the approximated price:

$$
C^{(k)}(y)=\sum_{l=0}^{k} \sum_{R_{l}} \frac{D^{L} C\left(y^{*}\right)}{\prod_{k=1}^{d} l_{k} !} \prod_{k=1}^{d-1}\left(y^{(k+1)}-y^{*(k)}\right)^{l_{k}},
$$

where $L=\left(l_{1}, l_{2}, \ldots, l_{d-1}\right)$ and $R_{l}=\left\{L \in \mathbb{N}^{d-1} / l_{1}+l_{2}+\cdots+\right.$ $\left.l_{d-1}=l, 0 \leq l_{k} \leq l\right\}$.

Taking into account Proposition 1 , a natural $k$ th-order Taylor approximation around $y^{*}=\left(y_{2}^{*}, y_{3}^{*}, \ldots, y_{d-1}^{*}\right)$ of 
the price $C_{B}$ of a basket option with payoff $h\left(S_{T}\right)$, defined as $C_{B}^{(k)}:=e^{-r T} E_{Q}\left[C^{(k)}\left(\tilde{Y}_{T}\right)\right]$, is obtained by

$$
\begin{aligned}
& C_{B}^{(k)}\left(y^{*}\right)=w_{1} \sum_{l=0}^{k} \sum_{R_{l}} \frac{D^{L} C\left(y^{*}\right)}{l_{1} ! l_{2} ! \cdots l_{d-1} !} \\
& \cdot E_{Q}\left[\exp \left(-\left(r-\frac{1}{2} \sigma_{Y_{T}^{(1)} / \widetilde{Y}_{T}}^{2}\right) T+\mu_{Y_{T}^{(1)} / \widetilde{Y}_{T}}\right)\right. \\
& \left.\quad \cdot \prod_{k=1}^{d-1}\left(Y_{T}^{(k+1)}-y^{*(k)}\right)^{l_{k}}\right]
\end{aligned}
$$

after replacing (16) into the expression for $C_{B}$ in the proposition above.

Remark 3. Notice that the approximation $C_{B}^{(k)}$ depends only on the derivatives of the function $C(y)$ with respect to $y$, which turns out to be the Black-Scholes price composed with the function $K(y)$, and the mixed exponential-power moments of a Gaussian multivariate distribution.

Remark 4. Sensitivities to the parameters can be computed by a similar approximation, as Greeks for a Black-Scholes option model are known. For example, the Delta with respect to the $j$ th asset can be approximated by

$$
\begin{aligned}
\Delta_{k}^{(j)} & =w_{1} \sum_{l=0}^{k} \sum_{R_{l}} \frac{D^{L}\left(\partial C\left(y^{*}\right) / \partial s^{(j)}\right)}{l_{1} ! l_{2} ! \cdots l_{d-1} !} E_{Q}[e \\
& \cdot \exp \left(-\left(r-\frac{1}{2} \sigma_{Y_{T}^{(1)} / \tilde{Y}_{T}}^{2}\right) T+\mu_{Y_{T}^{(1)} / \widetilde{Y}_{T}}\right) \\
& \left.\cdot \prod_{k=2}^{d}\left(Y_{T}^{(k)}-y_{k}^{*}\right)^{l_{k}}\right] .
\end{aligned}
$$

\section{Pricing Spreads Options by Taylor Approximations}

In order to illustrate the method studied in the previous section, we consider the case of a bidimensional spread option under the model given by (1) and (2) with covariance matrix:

$$
\Sigma=\left(\begin{array}{cc}
\sigma_{1}^{2} & \rho \sigma_{1} \sigma_{2} \\
\rho \sigma_{1} \sigma_{2} & \sigma_{2}^{2}
\end{array}\right)
$$

Notice that, for convenience, we have slightly changed some notations.
From (11), the conditional distribution of $Y_{T}^{(1)}$ given $Y_{T}^{(2)}$ is normal with mean and variance given by

$$
\begin{aligned}
\mu\left(Y_{T}^{(2)}\right):= & \mu_{Y_{T}^{(1)}} \tilde{Y}_{T} \\
= & r\left(1-\frac{\sigma_{1}}{\sigma_{2}} \rho\right) T+\frac{1}{2} \sigma_{1}\left(\sigma_{2} \rho-\sigma_{1}\right) T \\
& +\frac{\sigma_{1}}{\sigma_{2}} \rho Y_{T}^{(2)}, \\
\sigma:= & \sigma_{Y_{T}^{(1)} / \widetilde{Y}_{T}}=\sqrt{\left(1-\rho^{2}\right)} \sigma_{1} .
\end{aligned}
$$

Thus, we can write

$$
Y_{T}^{(1)}=\mu\left(Y_{T}^{(2)}\right)+\sqrt{T} \sigma Z \text { in law, }
$$

where $Z \sim N(0,1)$ independent of $Y_{T}$.

The $k$ th-order Taylor approximation in this case simplifies to

$$
\begin{aligned}
& C_{B}^{(k)}\left(y^{*}\right) \\
& \quad=\sum_{l=0}^{k} \frac{D^{l} C\left(y^{*}\right)}{l !} E_{Q}\left[\exp \left(-\left(r-\frac{1}{2} \sigma^{2}\right) T+\mu\left(Y_{T}^{(2)}\right)\right)\right. \\
& \left.\quad \cdot\left(Y_{T}^{(2)}-y^{*}\right)^{l}\right] .
\end{aligned}
$$

Moreover,

$$
\begin{aligned}
E_{Q} & {\left[\exp \left(\left(-r+\frac{1}{2} \sigma^{2}\right) T+\mu\left(Y_{T}^{(2)}\right)\right)\left(Y_{T}^{(2)}-y^{*}\right)^{l}\right] } \\
& =e^{A} E_{Q}\left[\exp \left(\frac{\sigma_{1}}{\sigma_{2}} \rho Y_{T}^{(2)}\right)\left(Y_{T}^{(2)}-y^{*}\right)^{l}\right],
\end{aligned}
$$

where

$$
A=-\left(\frac{1}{2} \rho^{2} \sigma_{1}^{2}+r \frac{\sigma_{1}}{\sigma_{2}} \rho-\frac{1}{2} \sigma_{1} \sigma_{2} \rho\right) T .
$$

Now, marginals of a multivariate normal distribution are normal too. Hence, we have that $Y_{T}^{(2)} \sim N\left(\left(r-(1 / 2) \sigma_{2}^{2}\right) T, T \sigma_{2}^{2}\right)$. The exponential-power moments can be calculated as

$$
\begin{aligned}
E_{Q} & {\left[\exp \left(\frac{\sigma_{1}}{\sigma_{2}} \rho Y_{T}^{(2)}\right)\left(Y_{T}^{(2)}-y^{*}\right)^{l}\right] } \\
= & \exp \left(\frac{\sigma_{1}}{\sigma_{2}} \rho\left(r-\frac{1}{2} \sigma_{2}^{2}\right) T\right) \\
& \cdot \sum_{m=0}^{l}\left(\begin{array}{c}
l \\
m
\end{array}\right)\left(\sqrt{T} \sigma_{2}\right)^{m} B\left(y^{*}\right)^{l-m} D^{m} M_{Z}\left(\sqrt{T} \sigma_{1} \rho\right)
\end{aligned}
$$

with

$$
B\left(y^{*}\right)=\left(r-\frac{1}{2} \sigma_{2}^{2}\right) T-y^{*}
$$


Next, integrating by parts,

$$
\begin{aligned}
D^{m} M_{Z}\left(\sqrt{T} \sigma_{1} \rho\right)=\exp \left(\frac{\sigma_{1}^{2} \rho^{2} T}{2}\right) \\
\quad \cdot \sum_{\nu=0}^{[m / 2]}\left(\begin{array}{c}
m \\
2 v
\end{array}\right)\left(\sigma_{1} \rho \sqrt{T}\right)^{m-2 v}(2 v-1) ! !,
\end{aligned}
$$

where $n ! !$ is the double factorial defined as the product of all odd numbers between 1 and $n$ including both. When the set is empty, by convention, the product is equal to one.

As a consequence, for $y^{*}=E_{Q}\left(Y_{T}^{(2)}\right)$, we have

$$
\begin{aligned}
& E_{Q}\left[\exp \left(\frac{\sigma_{1}}{\sigma_{2}} \rho Y_{T}^{(2)}\right)\left(Y_{T}^{(2)}-y^{*}\right)^{l}\right]=T^{l / 2} \sigma_{2}^{l} \\
& \cdot \exp \left(\frac{\sigma_{1}}{\sigma_{2}} \rho\left(r-\frac{1}{2} \sigma_{2}^{2}\right) T\right) \exp \left(\frac{\sigma_{1}^{2} \rho^{2} T}{2}\right) \\
& \cdot \sum_{v=0}^{l}\left(\begin{array}{l}
l \\
v
\end{array}\right)\left(\sigma_{1} \rho \sqrt{T}\right)^{l-v} E\left(Z^{v}\right) \\
& =T^{l / 2} \sigma_{2}^{l} e^{-A} \sum_{\nu=0}^{[l / 2]}\left(\begin{array}{c}
l \\
2 \nu
\end{array}\right)\left(\sigma_{1} \rho \sqrt{T}\right)^{l-2 v}(2 v-1) ! ! .
\end{aligned}
$$

After gathering all pieces and substituting in (22), we have the following result.

Proposition 5. The kth-order Taylor approximation of a spread contract with maturity at $T$ and strike price $K$, under the model described by (1) and (2), is given by

$$
\begin{aligned}
& C_{B}^{(k)}\left(y^{*}\right) \\
& =\sum_{l=0}^{k} \sum_{m=0}^{l} \frac{D^{l} C\left(y^{*}\right)}{l !}\left(\begin{array}{c}
l \\
m
\end{array}\right)\left(\sqrt{T} \sigma_{2}\right)^{m} B\left(y^{*}\right)^{l-m} E(m)
\end{aligned}
$$

with

$$
E(m)=\sum_{v=0}^{m}\left(\begin{array}{l}
m \\
v
\end{array}\right)\left(\sigma_{1} \rho \sqrt{T}\right)^{m-v} E_{Q}\left(Z^{v}\right)
$$

for $m=1,2, \ldots, k$ and $E(0)=1$, where $E_{Q} Z^{\nu}=(\nu-1) ! !$ if $\nu$ is even or zero if it is odd, and

$$
\begin{gathered}
K(y)=e^{-A}\left(K \exp \left(-\frac{\sigma_{1}}{\sigma_{2}} \rho y\right)\right. \\
\left.+S_{0}^{(2)} \exp \left(\left(1-\frac{\sigma_{1}}{\sigma_{2}} \rho\right) y\right)\right) .
\end{gathered}
$$

We now need to compute the derivatives of the function $C(y)$ with respect to $y$. By a straightforward calculation from Black-Scholes formula,

$$
\begin{aligned}
& D^{1} K(y)=e^{-A}\left(-\frac{\sigma_{1}}{\sigma_{2}} \rho K \exp \left(-\frac{\sigma_{1}}{\sigma_{2}} \rho y\right)\right. \\
& \left.+S_{0}^{(2)}\left(1-\frac{\sigma_{1}}{\sigma_{2}} \rho\right) \exp \left(\left(1-\frac{\sigma_{1}}{\sigma_{2}} \rho y\right)\right)\right), \\
& D^{2} K(y)=e^{-A}\left(\left(\frac{\sigma_{1}}{\sigma_{2}} \rho\right)^{2} K \exp \left(-\frac{\sigma_{1}}{\sigma_{2}} \rho y\right)\right. \\
& \left.+S_{0}^{(2)}\left(1-\frac{\sigma_{1}}{\sigma_{2}} \rho\right)^{2} \exp \left(\left(1-\frac{\sigma_{1}}{\sigma_{2}} \rho\right) y\right)\right) .
\end{aligned}
$$

Also,

$$
\begin{aligned}
D^{1} C & (y) \\
= & S_{0}^{(1)} f_{Z}\left(d_{1}(K(y))\right) D^{(1)} d_{1}(K(y)) \\
& -e^{-r T} D^{1} K(y) N\left(d_{2}(K(y))\right) \\
& -e^{-r T} K(y) f_{Z}\left(d_{2}(K(y))\right) D^{1} d_{1}(K(y)) \\
= & -\frac{D^{(1)} K(y)}{K(y) \sigma \sqrt{T}} A_{2}(y),
\end{aligned}
$$

where $f_{Z}$ is the density function of a standard normal random variable and

$$
\begin{aligned}
A_{2}(y)= & S_{0}^{(1)} f_{Z}\left(d_{1}(K(y))\right) \\
& +\sigma \sqrt{T} e^{-r T} K(y) N\left(d_{2}(K(y))\right) \\
& -e^{-r T} K(y) f_{Z}\left(d_{2}(K(y))\right) .
\end{aligned}
$$

Similarly, the second derivative is obtained as

$D^{2} C(y)$

$$
\begin{aligned}
& =-\frac{1}{\sigma \sqrt{T}}\left[A_{2}(y) \frac{K(y) D^{(2)} K(y)-\left(D^{1} K(y)\right)^{2}}{K^{2}(y)}\right. \\
& \left.+D^{(1)} A_{2}(y) \frac{D^{(1)} K(y)}{K(y)}\right]
\end{aligned}
$$

with

$$
\begin{aligned}
& D^{1} A_{2}(y) \\
& \quad=\frac{D^{1} K(y)}{K(y) \sigma \sqrt{T}}\left[S_{0}^{(1)} f_{Z}\left(d_{1}(K(y))\right) d_{1}(K(y))\right. \\
& +\sigma^{2} T e^{-r T} K(y) N\left(d_{2}(K(y))\right) \\
& -2 \sigma \sqrt{T} e^{-r T} K(y) f_{Z}\left(d_{2}(K(y))\right) \\
& -e^{-r T} K(y) f_{Z}\left(d_{2}(K(y))\right) d_{2}(K(y)) \\
& \left.+e^{-r T} \sigma \sqrt{T} K^{2}(y) f_{Z}\left(d_{2}(K(y))\right)\right] .
\end{aligned}
$$


In particular, when we expand the price around the point $y_{\text {mean }}=E_{\mathbb{Q}}\left(Y_{T}^{(2)}\right)=\left(r-(1 / 2) \sigma_{2}^{2}\right) T$ we have the first and second approximations given, respectively, by

$$
\begin{aligned}
C^{(1)}\left(y_{\text {mean }}\right)= & C\left(y_{\text {mean }}\right)+\sigma_{1} \sigma_{2} \rho T D^{1} C\left(y_{\text {mean }}\right) \\
C^{(2)}\left(y_{\text {mean }}\right)= & \widehat{p}_{1} \\
& +\frac{1}{2}\left[T \sigma_{2}^{2}\left(1+\sigma_{1}^{2} \rho^{2} T\right)\right] D^{2} C\left(y_{\text {mean }}\right)
\end{aligned}
$$

More generally, expanding around $y^{*} \in \mathbb{R}$, the first two approximations denoted by $\widehat{p}_{1}\left(y^{*}\right)$ and $\widehat{p}_{2}\left(y^{*}\right)$, respectively, are given by

$$
\begin{aligned}
& C_{B}^{(1)}\left(y^{*}\right)=C\left(y^{*}\right)+D^{1} C\left(y^{*}\right)\left(B\left(y^{*}\right)+\sqrt{T} \sigma_{2} E(1)\right)=C\left(y^{*}\right)+D^{1} C\left(y^{*}\right) \\
& \cdot\left(B\left(y^{*}\right)+T \sigma_{1} \sigma_{2} \rho\right), \\
& C^{(2)}\left(y^{*}\right)=C_{B}^{(1)}\left(y^{*}\right)+\frac{1}{2} D^{2} C\left(y^{*}\right) \\
& \cdot\left[B^{2}\left(y^{*}\right)+2 T \sigma_{1} \sigma_{2} \rho B\left(y^{*}\right)+T \sigma_{2}^{2}\left(1+T \sigma_{1}^{2} \rho^{2}\right)\right] .
\end{aligned}
$$

\section{Approximation by Chebyshev Polynomials}

We study an alternative approximation of the price via Chebyshev polynomials. For definition and their basic properties see, for example, [17].

Denoting by $\left(T_{k}(y)\right)_{k \in \mathbb{N}}$ the sequence of Chebyshev polynomials of first type on $[-1,1]$, we consider the $n$th approximation of the function $C(y)$ on the interval $[a, b]$ described by equation in terms of Chebyshev polynomials, the one given by

$$
\begin{aligned}
C_{\mathrm{CH}}(y, a, b, n)= & \frac{1}{2} \widehat{c}_{0} 1_{[a, b]}(y) \\
& +\sum_{k=1}^{n} \widehat{c}_{k} T_{k}^{a, b}(y) 1_{[a, b]}(y),
\end{aligned}
$$

where $\left(T_{k}^{a, b}(x)\right)_{k \in \mathbb{N}}$ is the sequence of Chebyshev polynomials of first type on $[a, b]$ defined by

$$
T_{k}^{a, b}(y)=T_{k}\left(-1+\frac{2}{(b-a)}(y-a)\right), \quad a \leq y \leq b,
$$

and the values $\left(\widehat{c}_{k}\right)_{0 \leq k \leq N}$ are estimators of the corresponding coefficients in the Chebyshev expansion.
Chebyshev polynomials on $[a, b]$ are orthogonal with respect to the scalar product defined as

$$
\langle f, g\rangle=\int_{a}^{b} f(x) g(x) w_{a, b}(x) d x
$$

with weight function $w_{a, b}(x)=\left(1-(2(x-a) /(b-a)-1)^{2}\right)^{-1 / 2}$. Notice that

$$
\left\|T_{k}^{a, b}\right\|^{2}= \begin{cases}\frac{(b-a) \pi}{4} & \text { for } k \neq 0 \\ \frac{(b-a) \pi}{2} & \text { for } k=0 .\end{cases}
$$

Then, for $k \neq 0$ the coefficients in the expansion can be calculated as

$$
\begin{aligned}
c_{k} & =\frac{\left\langle C, T_{k}^{a, b}\right\rangle}{\left\|T_{k}^{a, b}\right\|^{2}}=\frac{4}{(b-a) \pi} \\
& \cdot \int_{a}^{b} C(y) T_{k}^{a, b}(y) w_{a, b}(y) d y=\frac{4}{(b-a) \pi} \\
& \cdot \int_{a}^{b} C(y) T_{k}\left(-1+\frac{2}{(b-a)}(y-a)\right) w_{a, b}(y) d y \\
& =\frac{2}{\pi} \int_{-1}^{1} C\left(a+\frac{b-a}{2}(x+1)\right) T_{k}(x) w_{-1,1}(x) d x \\
& =\frac{2}{\pi} \int_{0}^{\pi} C\left(a+\frac{b-a}{2}(\cos \theta+1)\right) \cos (k \theta) d \theta
\end{aligned}
$$

after changes of variables $x=-1+(2 /(b-a))(y-a)$ and $x=\cos \theta$.

Also,

$$
c_{0}=\frac{1}{\pi} \int_{0}^{\pi} C\left(a+\frac{b-a}{2}(\cos \theta+1)\right) d \theta .
$$

From the trapezoidal rule to approximate Riemann integrals, the coefficients $\left(c_{k}\right)_{0 \leq k \leq N}$ can be estimated by an equidistant partition of $N$ points on $[0, \pi]$.

Chebyshev polynomials of first type can be written in terms of powers of the variable. From [18],

$$
T_{k}^{a, b}(x)=\sum_{l=0}^{[k / 2]} b_{l}^{(k)}\left(-1+\frac{2}{b-a}(x-a)\right)^{k-2 l},
$$


where

$$
\begin{aligned}
& b_{l}^{(k)} \\
& = \begin{cases}(-1)^{k / 2} & \text { for } l=\frac{k}{2} \text { and } k \text { even } \\
(-1)^{l} 2^{k-2 l-1} \frac{k}{k-l}\left(\begin{array}{c}
k-l \\
l
\end{array}\right) & \text { for } l<\frac{k}{2}\end{cases}
\end{aligned}
$$

In a similar way to the case of Taylor polynomials, define the $n$th order Chebyshev approximation for the basket option price as

$$
\begin{aligned}
& C_{\mathrm{CH}}(a, b, n) \\
& \quad:=w_{1} E_{Q}\left[\exp \left(-\left(r-\frac{1}{2} \sigma^{2}\right) T+\mu\left(Y_{T}^{(2)}\right)\right)\right. \\
& \left.\cdot \widetilde{C}_{\mathrm{CH}}\left(Y_{T}^{(2)}, a, b, n\right)\right] .
\end{aligned}
$$

The next theorem provides the Chebyshev approximation for the price of a basket option.

Theorem 6. The nth order Chebyshev's approximation of the price $C_{B}$ of a European Basket option under the model given by (1) and (2) is given by

$$
\begin{aligned}
C_{C H}(a, b, n)=\frac{w_{1}}{2} \\
\cdot \widehat{c}_{0}\left[N\left(\widetilde{b}-\sigma_{1} \rho \sqrt{T}\right)-N\left(\tilde{a}-\sigma_{1} \rho \sqrt{T}\right)\right]+w_{1} \\
\cdot \exp \left(-\frac{1}{2} \rho^{2} \sigma_{1}^{2} T\right) \\
\quad \cdot \sum_{k=1}^{n} \sum_{l=0}^{[k / 2]} \widehat{c}_{k} b_{l}^{(k)}\left(\frac{2 \sigma_{2} \sqrt{T}}{b-a}\right)^{k-2 l} \widehat{G}(k-2 l),
\end{aligned}
$$

where

$$
\begin{aligned}
\widehat{G}(k) & =\sum_{m=0}^{k}\left(\begin{array}{c}
k \\
m
\end{array}\right)\left(\frac{2\left(r-(1 / 2) \sigma_{2}^{2}\right) T-a-b}{2 \sigma_{2} \sqrt{T}}\right)^{m} \\
\cdot & \left.\frac{d^{k-m} M_{Z}(u, \tilde{a}, \tilde{b})}{d u^{k-m}}\right|_{u=\sigma_{1} \rho \sqrt{T}}
\end{aligned}
$$

for $k=0,1,2, \ldots, n$,

$$
\begin{aligned}
& \tilde{b}=\frac{b-\left(r-(1 / 2) \sigma_{2}^{2}\right) T}{\sigma_{2} T}, \\
& \tilde{a}=\frac{a-\left(r-(1 / 2) \sigma_{2}^{2}\right) T}{\sigma_{2} T} .
\end{aligned}
$$

Proof. Notice that

$$
\begin{gathered}
e^{A} E_{\mathscr{Q}}\left[\exp \left(\frac{\sigma_{1}}{\sigma_{2}} \rho Y_{T}^{(2)}\right) 1_{(-\infty, b)}\left(Y_{T}^{(2)}\right)\right]=e^{A} \\
\cdot \exp \left(\left(r-\frac{1}{2} \sigma_{2}^{2}\right) \frac{\sigma_{1}}{\sigma_{2}} \rho T\right) \\
\cdot E_{\mathscr{Q}}\left[\exp \left(\sigma_{1} \rho \sqrt{T} Z\right) 1_{(-\infty, \tilde{b})}(Z)\right]=e^{A} \\
\cdot \exp \left(\left(r-\frac{1}{2} \sigma_{2}^{2}\right) \frac{\sigma_{1}}{\sigma_{2}} \rho T+\frac{1}{2} \sigma_{1}^{2} \rho^{2} T\right) \frac{1}{\sqrt{2 \pi}} \\
\cdot \int_{-\infty}^{\tilde{b}-\sigma_{1} \rho \sqrt{T}} \exp \left(-\frac{1}{2} z^{2}\right) d z=N\left(\tilde{b}-\sigma_{1} \rho \sqrt{T}\right) .
\end{gathered}
$$

Hence,

$$
\begin{aligned}
& e^{A} E_{Q}\left[\exp \left(\frac{\sigma_{1}}{\sigma_{2}} \rho Y_{T}^{(2)}\right) 1_{[a, b]}\left(Y_{T}^{(2)}\right)\right] \\
& =N\left(\widetilde{b}-\sigma_{1} \rho \sqrt{T}\right)-N\left(\widetilde{a}-\sigma_{1} \rho \sqrt{T}\right) .
\end{aligned}
$$

From Proposition 1, we take into account (39) and (45) to get

$$
\begin{aligned}
& C_{\mathrm{CH}}(a, b, n)=w_{1} e^{A} E_{Q}\left(\exp \left(\frac{\sigma_{1}}{\sigma_{2}} \rho Y_{T}^{(2)}\right)\right. \\
& \left.\cdot C_{\mathrm{CH}}\left(Y_{T}^{(2)}, a, b, n\right)\right)=\frac{w_{1}}{2} \widehat{c}_{0}\left[N\left(\widetilde{b}-\sigma_{1} \rho \sqrt{T}\right)\right. \\
& \left.-N\left(\widetilde{a}-\sigma_{1} \rho \sqrt{T}\right)\right] \\
& +w_{1} e^{A} \sum_{k=1}^{n} \widehat{c}_{k} E_{Q}\left[\exp \left(\frac{\sigma_{1}}{\sigma_{2}} \rho Y_{T}^{(2)}\right) T_{k}^{a, b}\left(Y_{T}^{(2)}\right)\right. \\
& \left.\cdot 1_{[a, b]}\left(Y_{T}^{(2)}\right)\right]=\frac{w_{1}}{2} \widehat{c}_{0}\left[N\left(\widetilde{b}-\sigma_{1} \rho \sqrt{T}\right)-N(\widetilde{a}\right. \\
& \left.\left.-\sigma_{1} \rho \sqrt{T}\right)\right]+w_{1} e^{A} \sum_{k=1}^{n} \sum_{l=0}^{[k / 2]} \frac{\widehat{c}_{k} b_{l}^{(k)}}{(b-a)^{k-2 l}} \\
& \cdot E_{Q}\left[\exp \left(\frac{\sigma_{1}}{\sigma_{2}} \rho Y_{T}^{(2)}\right)\left(2 Y_{T}^{(2)}-a-b\right)^{k-2 l}\right. \\
& \left.\cdot 1_{[a, b]}\left(Y_{T}^{(2)}\right)\right] \cdot
\end{aligned}
$$


Now,

$$
\begin{aligned}
& E_{Q}\left[\exp \left(\frac{\sigma_{1}}{\sigma_{2}} \rho Y_{T}^{(2)}\right)\left(2 Y_{T}^{(2)}-a-b\right)^{k-2 l} 1_{[a, b]}\left(Y_{T}^{(2)}\right)\right] \\
& =\sum_{m=0}^{k-2 l}\left(\begin{array}{c}
k-2 l \\
m
\end{array}\right)\left(2 E_{Q}\left[Y_{T}^{(2)}\right]-a-b\right)^{m} \\
& \cdot 2^{k-2 l-m} E_{Q}\left[\exp \left(\frac{\sigma_{1}}{\sigma_{2}} \rho Y_{T}^{(2)}\right)\right. \\
& \left.\cdot\left(Y_{T}^{(2)}-E_{Q}\left[Y_{T}^{(2)}\right]\right)^{k-2 l-m} 1_{[a, b]}\left(Y_{T}^{(2)}\right)\right]=\exp \left(\frac{\sigma_{1}}{\sigma_{2}}\right. \\
& \left.\cdot \rho\left(r-\frac{1}{2} \sigma_{2}^{2}\right) T\right)\left(2 \sigma_{2} \sqrt{T}\right)^{k-2 l} \\
& \cdot \sum_{m=0}^{k-2 l}\left(\begin{array}{c}
k-2 l \\
m
\end{array}\right)\left(\frac{2\left(r-(1 / 2) \sigma_{2}^{2}\right) T-a-b}{2 \sigma_{2} \sqrt{T}}\right)^{m} \\
& \cdot E_{Q}\left[\exp \left(\sigma_{1} \rho \sqrt{T} Z\right) Z^{k-2 l-m} 1_{[\tilde{a}, \tilde{b}]}(Z)\right]=\exp \left(\frac{\sigma_{1}}{\sigma_{2}}\right. \\
& \left.\cdot \rho\left(r-\frac{1}{2} \sigma_{2}^{2}\right) T\right)\left(2 \sigma_{2} \sqrt{T}\right)^{k-2 l} \\
& \cdot \sum_{m=0}^{k-2 l}\left(\begin{array}{c}
k-2 l \\
m
\end{array}\right)\left(\frac{2\left(r-(1 / 2) \sigma_{2}^{2}\right) T-a-b}{2 \sigma_{2} \sqrt{T}}\right)^{m} \\
& \left.\cdot \frac{d^{k-2 l-m} M_{Z}(u, \tilde{a}, \tilde{b})}{d u^{k-2 l-m}}\right|_{u=\sigma_{1} \rho \sqrt{T}} .
\end{aligned}
$$

Substituting the last expression into (53), we get (48).

\section{Numerical Results}

As a benchmark setting, we consider initial prices of $S_{0}^{(1)}=$ 100 and $S_{0}^{(2)}=96$ dollars, strike price of $K=1$ dollar, maturity at $T=1$ year, and an annual interest rate of $r=3 \%$ under a bivariate Black-Scholes model, with a negative correlation $\rho=-0.3$ and respective volatilities equal to $\sigma_{1}=0.3$ and $\sigma_{2}=0.1$.

In Figure 1, we show the conditional price function on $[-1,1]$ for the benchmark setting (blue line), together with the first and second Taylor expansions around the mean value of $Y_{T}^{(2)}$; that is, $E_{\mathscr{Q}}\left(Y_{T}^{(2)}\right)=\left(r-(1 / 2) \sigma_{2}^{2}\right) T$ (green and red lines, resp.). While the second-order approximation offers a reasonable local fit, significant errors may be found for values far from the mean. These errors may impact the price given by its conditional expected value under the risk neutral probability when, for example, volatilities are high.

Notice that, due to the concavity of function $C$, the first approximation underestimates the price. Not surprisingly, the second approximation estimates the price fairly well for values close to the point mean value while it is less accurate for values far from the mean. Although it seems as a drawback

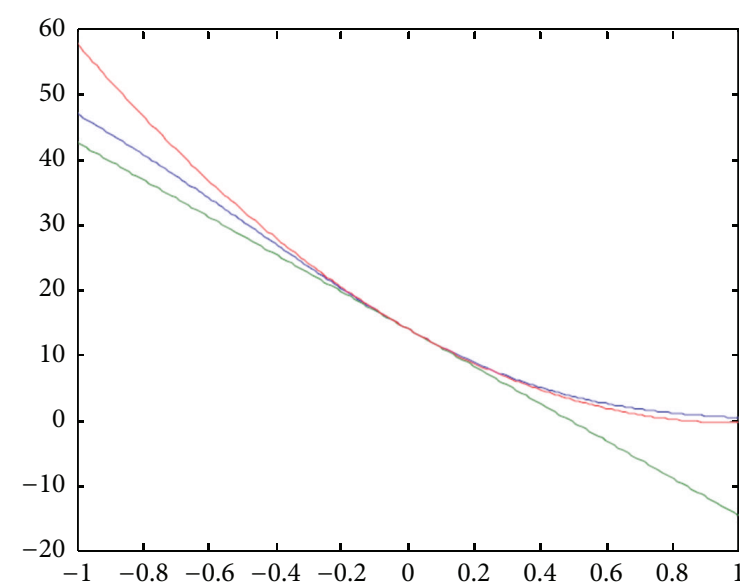

Figure 1: Approximations of the conditional price based on Taylor polynomials using the benchmark set of parameters. The blue line describes the conditional price on $[-1,1]$ while the green and red lines show first- and second-order expansions, respectively.

of the method, it does not constitute a serious problem as values far from the mean are infrequent, and thus the error in calculating the expected value from a Taylor approximation is fairly small.

A more promising result is obtained when an approximation of the conditional price is done via Chebyshev polynomials. Figure 2 represents, clockwise from top left, approximations of 4th, 10th, and 15th orders, respectively. Expansions of the 10th and 15th orders are practically indistinguishable from the original function. Coefficients in the expansion are calculated following a trapezoidal rule with 100 points on the interval $[-1.5,1.5]$.

In Table 1, we show the impact of correlation on the prices of spread contracts obtained under the benchmark parameter set. Prices are computed by a Monte Carlo approach based on 10 million simulations of the asset prices following a geometric bivariate Black-Scholes model with correlated Brownian motions (column 2). In addition, we implement a second-order Taylor expansion and an approximation by Chebyshev polynomials of order $n=15$. We consider positive, negative, large, moderated, and weak correlations. In all cases, the Chebyshev approximation shows a notable agreement with Monte Carlo prices at a lesser computational cost.

Notice that Chebyshev expansion requires extra quadrature to compute the polynomial coefficients as opposed to Taylor where we simply need to evaluate the corresponding derivatives. Hence, the computational complexity in the former multiplies by the number of points used in the trapezoid rule.

In Table 2, we show average routine times for the price calculation following Monte Carlo, Taylor, and Chebyshev expansions. The average is taken upon runs for different parameters in the parametric set. Routines were implemented on a standard PC using MATLAB language. Chebyshev approximation works about 200 times faster than the standard Monte Carlo approach. Taylor approach has an even 
TABLE 1: Spread prices for the benchmark parameters and several values of $\rho$, using Monte Carlo, a Taylor second-order approximation around $y^{*}=0$, and a Chebyshev approximation with 15 terms, $a=-4$ and $b=0.25$.

\begin{tabular}{lccc}
\hline Correlation & Monte Carlo & Taylor second approx. & Chebyshev, $n=15$ \\
\hline$\rho=-0.1$ & 14.2921 & 13.8709 & 14.2906 \\
$\rho=0.1$ & 13.56278 & 14.78882 & 13.5649 \\
$\rho=-0.3$ & 14.9734 & 15.0065 & 14.9629 \\
$\rho=0.3$ & 12.8085 & 12.7901 & 12.7903 \\
$\rho=-0.5$ & 15.6273 & 15.9238 & 15.6316 \\
$\rho=0.5$ & 11.9525 & 11.9646 & 11.9566 \\
$\rho=-0.7$ & 16.2421 & 17.5217 & 16.2521 \\
$\rho=0.7$ & 11.0315 & 11.1947 & 11.0529 \\
\hline
\end{tabular}
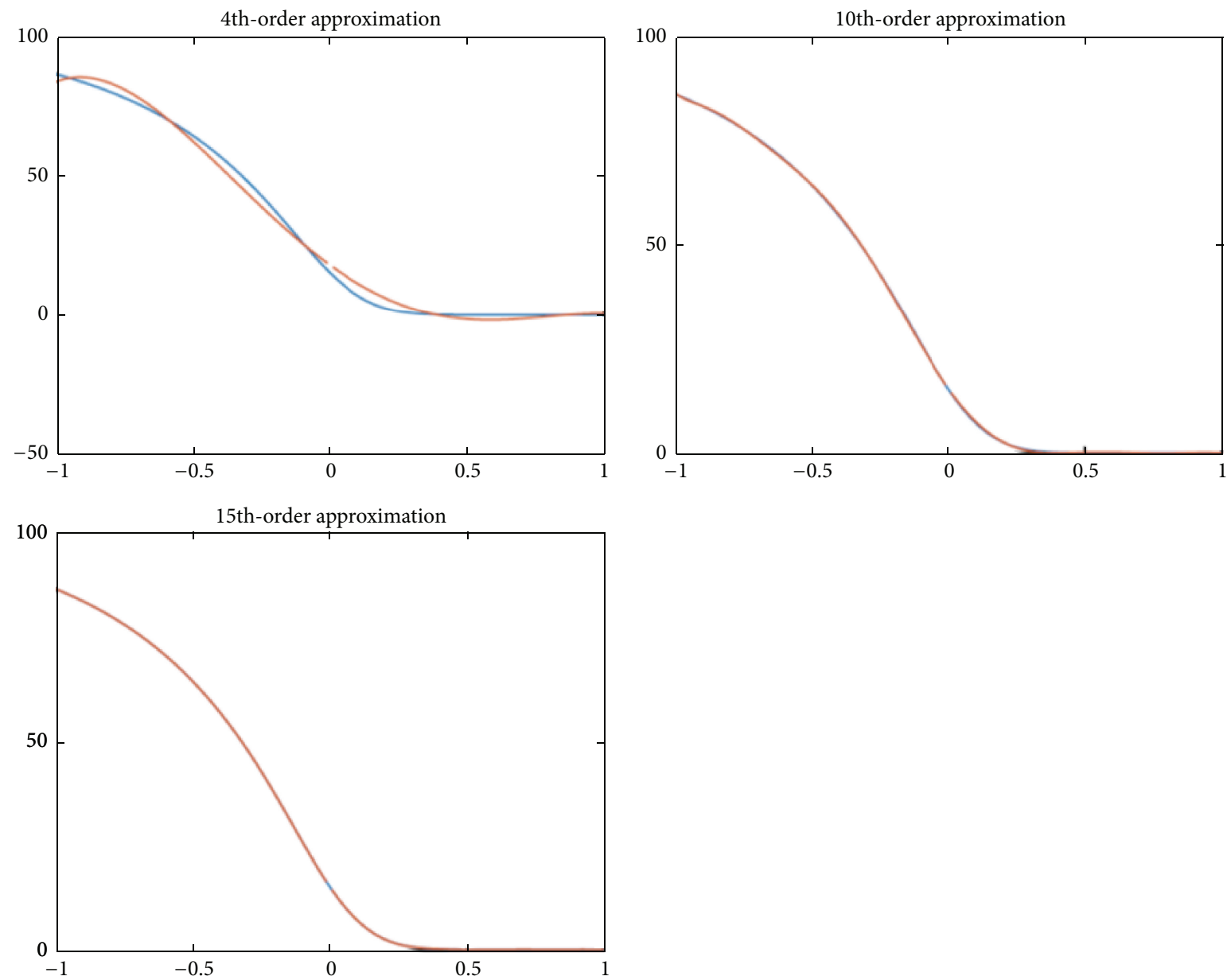

FIGURE 2: Approximations of the conditional price based on Chebyshev polynomials using the benchmark set of parameters. Clockwise, from top left are 4 th, 10 th, and 15 th orders, respectively. The truncation interval is $[-1.5,1.5]$.

lesser computational time, but for large asset correlations it is not as accurate as the former.

Due to the steepness of the function $C(y)$, the Chebyshev approximation is sensible to the truncation interval $[a, b]$. In our numerical computations, we have used $a=-4$ and $b=$ 0.25 . Within the range of parameter considered, most values of $Y_{T}^{(2)}$ lie on the selected interval $[a, b]$; hence, truncation does not affect the mixed exponential-power function by much. Otherwise, the approximation might be improved by taking $C(a)$ if $y<a$ and by $C(b)$ if $y>b$.

The selection of $a$ and $b$ is a delicate point. A priori, there is no theoretical limit in the length of the interval. Moreover, a large interval will result in a better approximation. On the flip side, the effort in the estimation of coefficients $c_{k}$ in 


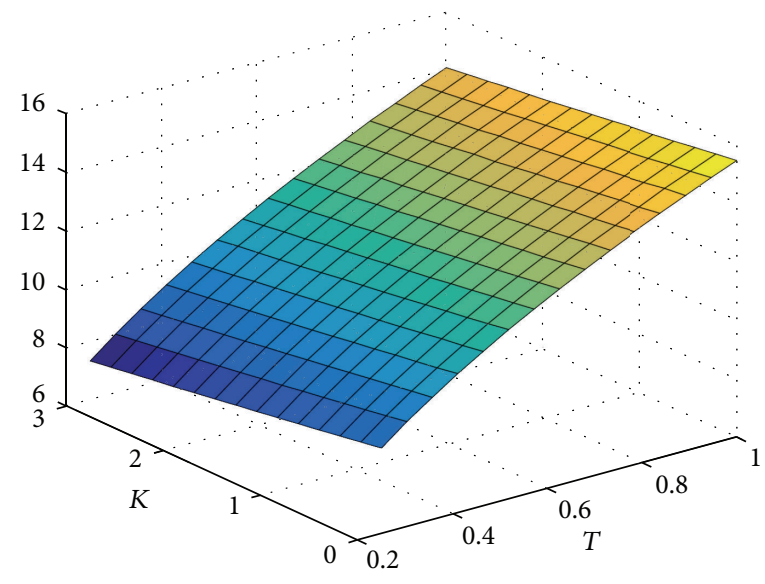

(a) Price computed by a Monte Carlo approach

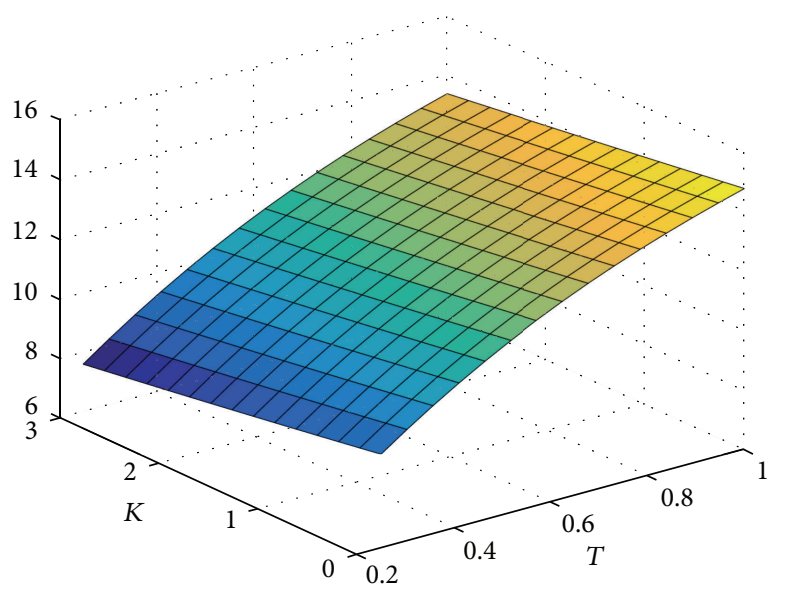

(b) Price computed by a Chebyshev expansion

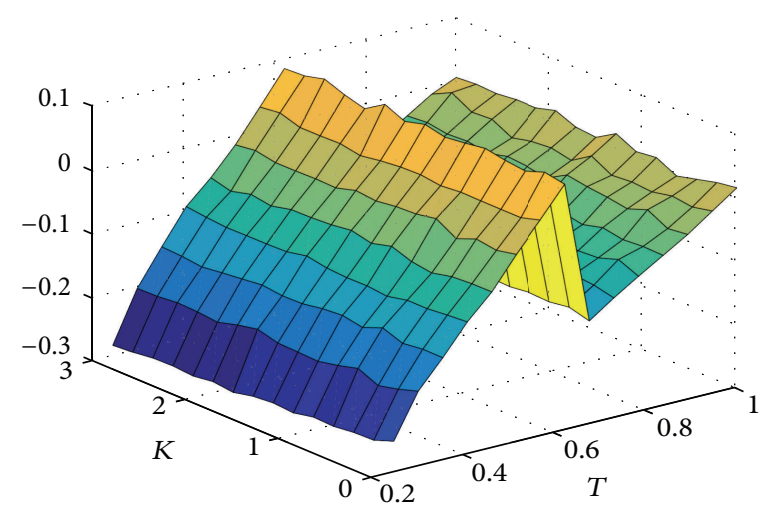

(c) Difference of prices between Monte Carlo and Chebyshev

FiguRE 3: Prices of a spread contract with maturity between one month and one year and strike price between 0 and 3 dollars under the benchmark parametric set. (c) Difference between prices obtained for both methods.

TABlE 2: Average routine times for Monte Carlo, Taylor, and Chebyshev approaches on a PC using MATLAB language.

\begin{tabular}{lcc}
\hline Monte Carlo & $\begin{array}{c}\text { Taylor second } \\
\text { approx. }\end{array}$ & Chebyshev, $n=15$ \\
\hline $2.048 \mathrm{sec}$. & $0.0076 \mathrm{sec}$. & $0.012 \mathrm{sec}$. \\
\hline
\end{tabular}

the polynomial expansion will increase for larger intervals. An intuitive criterion to select the truncation values might consist in looking at points where the function $C(y)$ flattens out.

On the other hand, if the second asset has a high volatility, the length of the truncating interval should increase to avoid having too many points outside the interval.

The method is stable for the number of points considered in the trapezoidal rule. Also, the approximation gets close to the actual price after a fairly moderate number of polynomials. For $n=10$, the method shows a good approximation within an error in the order of a penny. For $n=15$ and $n=20$ the approximation improves even more. For approximations of larger orders, the gain in precision does not compensate the increase in computational time.
Figure 3 shows prices of a spread contract based on a Chebyshev approximation of order $n=15$. Maturity times range from one month to one year, while strike prices go from zero (exchange option) to 3 dollars. Results are consistent with an increase in the contract prices with higher maturity and their decrease with the increase of the strike price. Figure 3(c) at the bottom, conveniently scaled, shows the difference between both prices. The average relative error is $0.0075 \%$.

Figure 4 shows the price of the contract as a function of volatilities of both underlying assets. For symmetry, we only have considered $\sigma_{1} \geq \sigma_{2}$ with volatilities ranging within $10 \%-50 \%$. Again, we can observe a remarkable agreement between prices obtained by Monte Carlo and Chebyshev approximation. As expected, the price of the spread increases with the increment in the volatility. The average relative error is $0.0023 \%$.

\section{Conclusions}

We compare three methods to price spreads options under a bivariate Black-Scholes model with correlated Brownian motions versus a standard Monte Carlo approach. Our results 


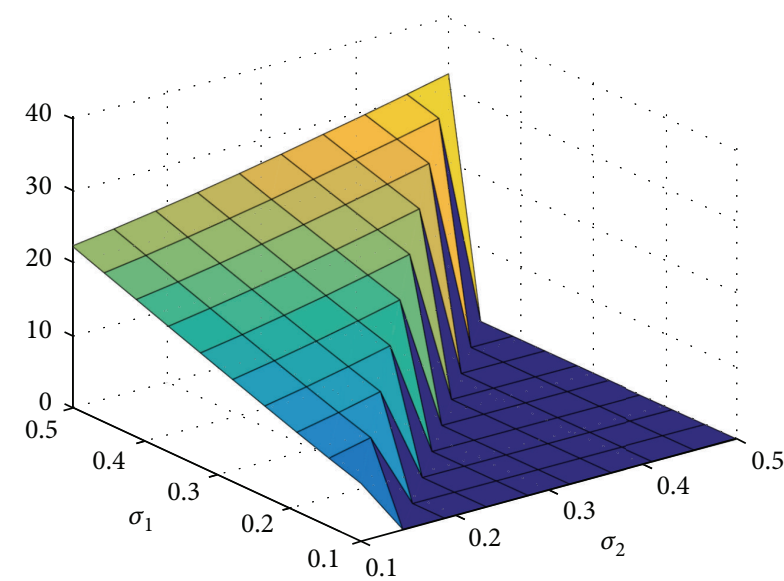

(a) Price computed by a Monte Carlo approach, $n=10^{7}$

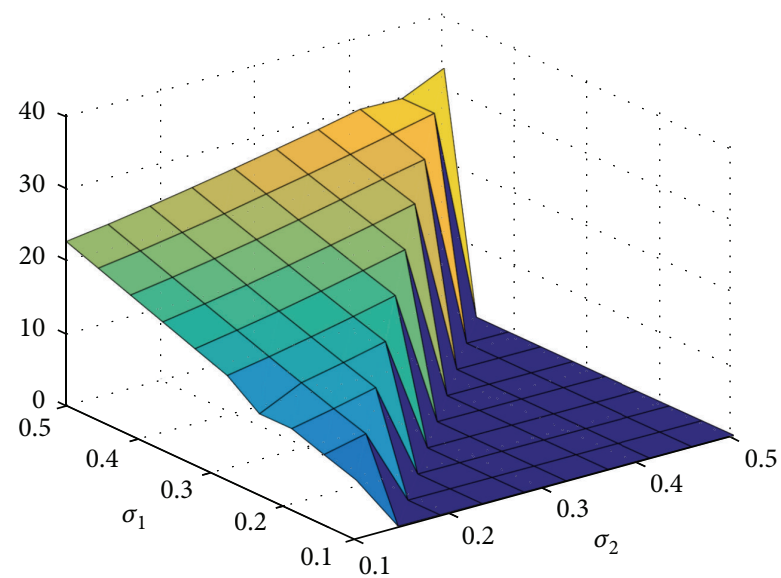

(b) Price computed by a Chebyshev expansion, order 15

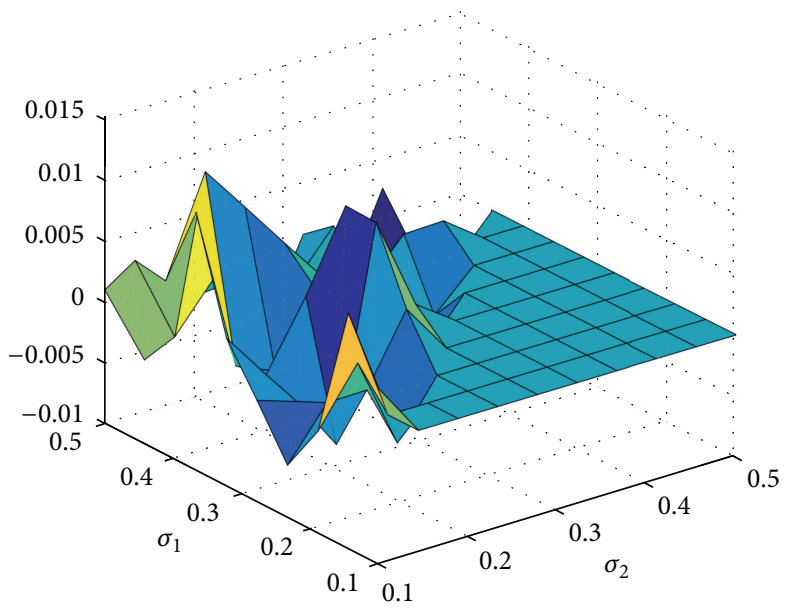

(c) Difference of prices between Monte Carlo and Chebyshev

Figure 4: Prices of a spread contract with log-return volatilities between $10 \%$ and $50 \%$ under the benchmark parametric set. (c) Difference between prices obtained for both methods. We consider, without loss of generality, the case $\sigma_{1} \geq \sigma_{2}$.

show that Taylor approximation does not offer a uniform convergence, and hence a poor result when values are far from the point around the expansion is taken. For some values in the parameter set, it may affect the corresponding expected value.

The approximation based on Chebyshev polynomials seems to be appropriate in terms of the balance offered between accuracy and computational cost. Moreover, the method is suitable to be implemented in more general models provided the conditional distribution is available.

\section{Competing Interests}

The authors declare that there is no conflict of interests regarding the publication of this manuscript.

\section{Acknowledgments}

Both authors would like to recognize NSERC for its support.

\section{References}

[1] N. D. Pearson, "An Efficient Approach for Pricing Spread Options," The Journal of Derivatives, vol. 3, no. 1, pp. 76-91, 1995.

[2] M. Li, S.-J. Deng, and J. Zhou, "Closed-form approximations for spread option prices and Greeks," Journal of Derivatives, vol. 15, no. 3, pp. 58-80, 2008.

[3] R. Pachon, "Numerical pricing of european options with arbitrary payoffs," SSRN Electronic Journal, 2016.

[4] M. Gass, K. Glau, M. Mahlstedt, and M. Mair, "Chebyshev interpolation for parametric option pricing," https://arxiv .org/abs/1505.04648.

[5] E. Kirk, Correlation in the Energy Markets, in Managing Energy Price Risk, Risk Publications and Enron, London, UK, 1995.

[6] R. Carmona and V. Durrleman, "Pricing and hedging spread options," SIAM Review, vol. 45, no. 4, pp. 627-685, 2003.

[7] S. Borokova, F. J. Permana, and H. Weide, "A closed form approach to the valuation and hedging of basket and spread options," The Journal of Derivatives, vol. 14, no. 4, pp. 8-24, 2009.

[8] A. Alvarez, M. Escobar, and P. Olivares, "Spread Options under Stochastic Covariance and Jumps. Pricing two dimensional 
derivatives under stochastic correlation," International Journal of Financial Markets and Derivatives, vol. 2, no. 4, pp. 265-287, 2011.

[9] A. Venkatramanan and C. Alexander, "Closed form approximations for spread options," Applied Mathematical Finance, vol. 18, no. 5, pp. 447-472, 2011.

[10] M. Li, J. Zhou, and S.-J. Deng, "Multi-asset spread option pricing and hedging," Quantitative Finance, vol. 10, no. 3, pp. 305-324, 2010.

[11] J. Hull and A. White, "The pricing of options on assets with stochastic volatilities," The Journal of Finance, vol. 42, no. 2, pp. 281-300, 1987.

[12] N. Ju, "Pricing Asian and basket options via Taylor expansion," The Journal of Computational Finance, vol. 5, no. 3, pp. 79-103, 2002.

[13] Q.-J. Meng and D. Ding, "An efficient pricing method for rainbow options based on two-dimensional modified sine-sine series expansions," International Journal of Computer Mathematics, vol. 90, no. 5, pp. 1096-1113, 2013.

[14] F. Fang and C. W. Oosterlee, "A novel pricing method for European options based on Fourier-cosine series expansions," SIAM Journal on Scientific Computing, vol. 31, no. 2, pp. 826848, 2008.

[15] W. Margrabe, "The value of an option to exchange one asset for another," The Journal of Finance, vol. 33, no. 1, pp. 177-186, 1978.

[16] Y. L. Tong, The Multivariate Normal Distribution, Springer, Berlin, Germany, 1989.

[17] J. C. Mason and D. C. Handscomb, Chebyshev Polynomials, CRC Press Company, Boca Raton, Fla, USA, 2003.

[18] A. Gil, J. Segura, and N. Temme, Numerical Methods for Special Functions, SIAM, Philadelphia, Pa, USA, 2007. 


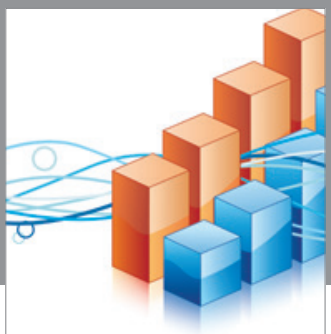

Advances in

Operations Research

vatem alat4

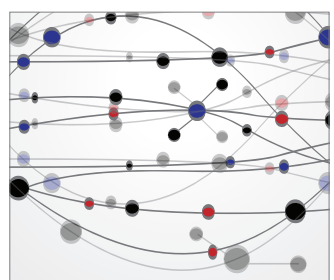

\section{The Scientific} World Journal
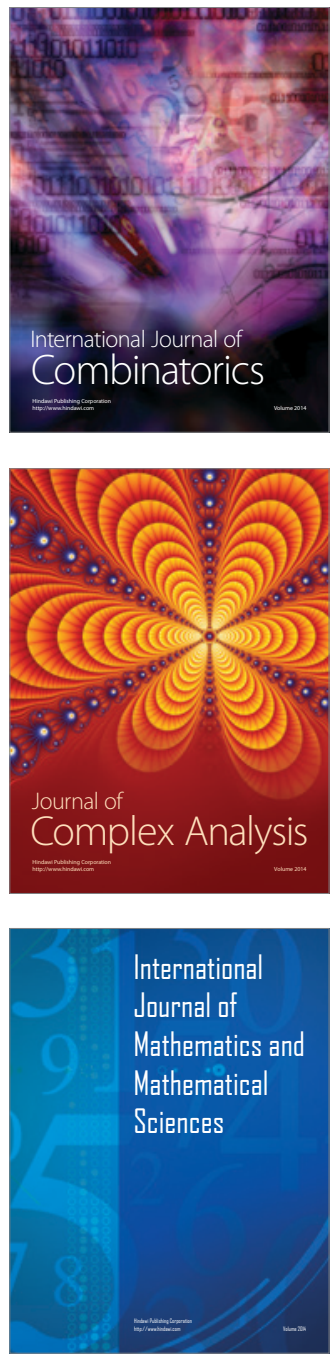
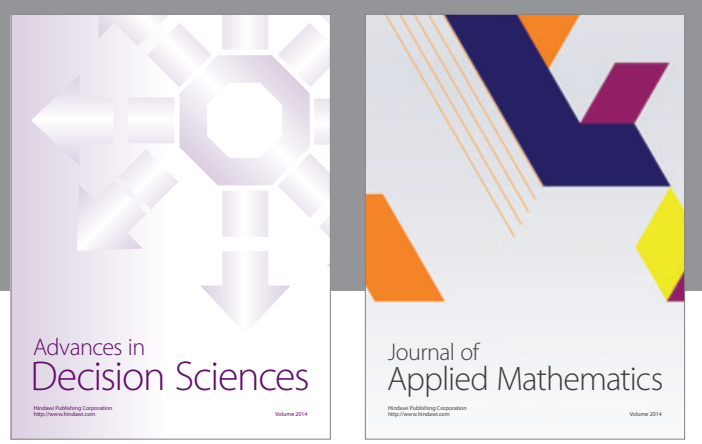

Algebra

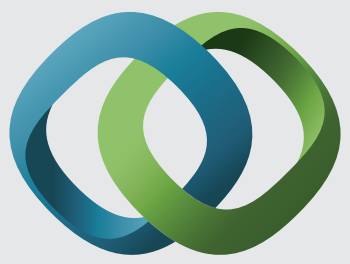

\section{Hindawi}

Submit your manuscripts at

http://www.hindawi.com
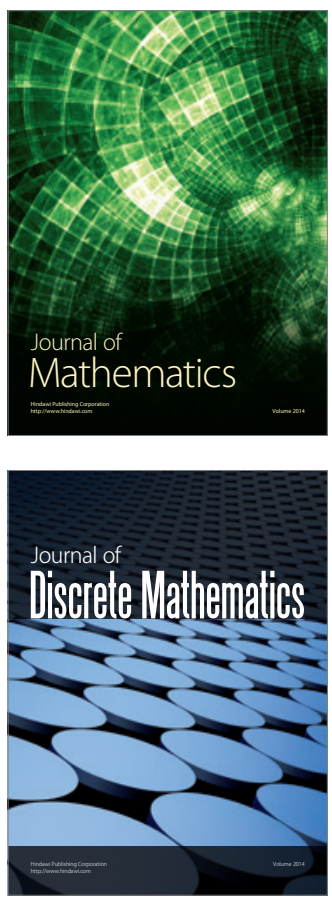

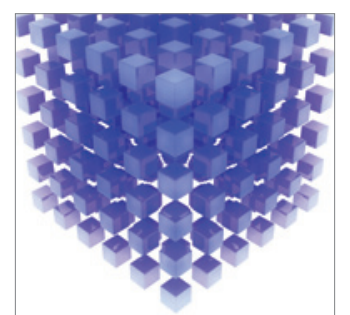

Mathematical Problems in Engineering
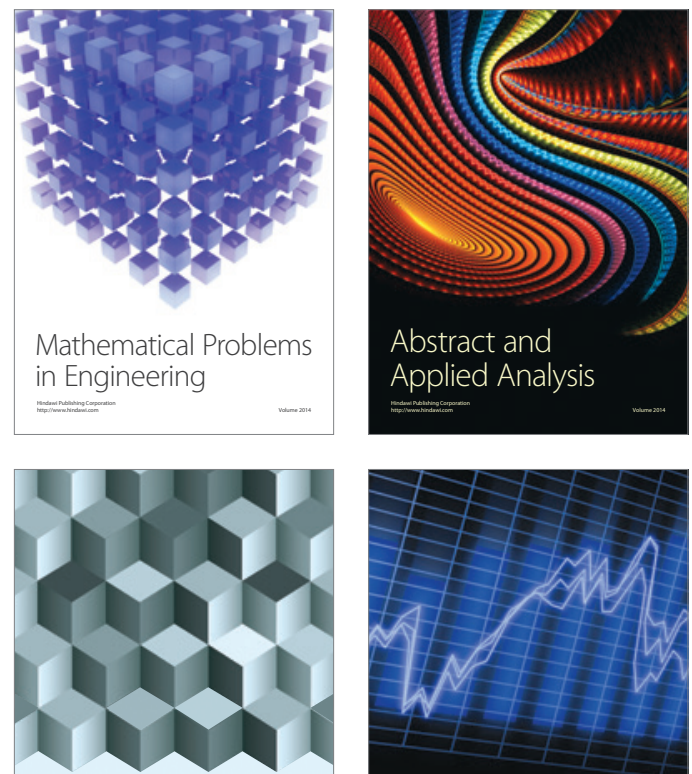

Journal of

Function Spaces

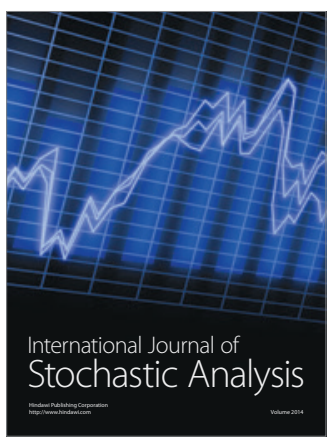

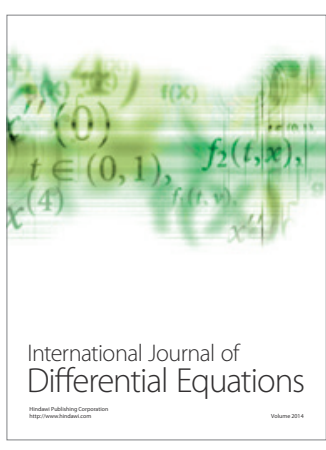
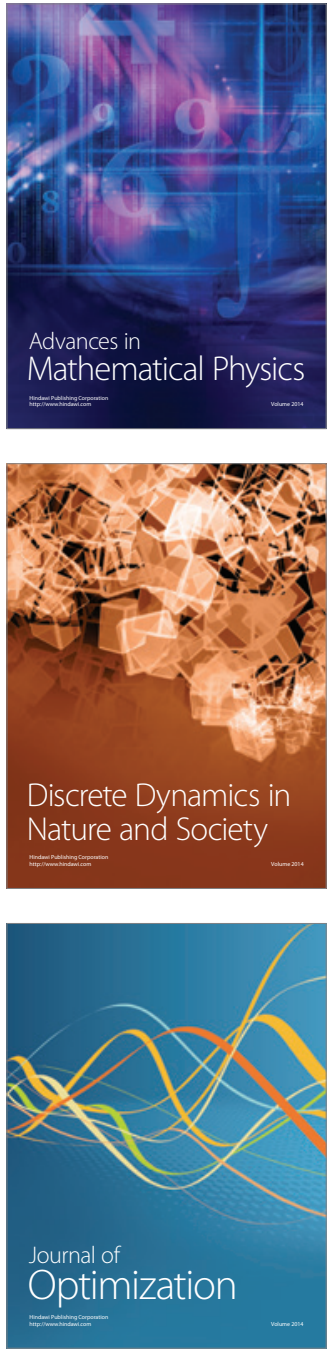